Originalien

Anaesthesist 2020 69:388-396

https://doi.org/10.1007/s00101-020-00772-z

Eingegangen: 14. Juni 2019

Überarbeitet: 9. März 2020

Angenommen: 29. März 2020

Online publiziert: 28. April 2020

(c) Der/die Autor(en) 2020

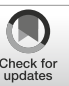

A. Rand ${ }^{1,2} \cdot$ C. A. Avila González ${ }^{1,5} \cdot$ G. C. Feigl ${ }^{3} \cdot$ T. Mäcken ${ }^{1}$ T. Weiß ${ }^{1,4} \cdot$ P. K. Zahn' $~$ R. J. Litz ${ }^{1,5}$

1 Universitätsklinik für Anästhesiologie, Intensiv-, Palliativ- und Schmerzmedizin, BG Universitätsklinikum Bergmannsheil GmbH der Ruhr Universität Bochum, Bochum, Deutschland

${ }^{2}$ Klinik für Anästhesiologie und Intensivmedizin, Universitätsklinikum Carl Gustav Carus, TU Dresden, Dresden, Deutschland

${ }^{3}$ Institut für Makroskopische und klinische Anatomie, Medizinische Universität Graz, Graz, Österreich

${ }^{4}$ Klinik für Anästhesie und Intensivmedizin, Kantonspital Thurgau, Münsterlingen, Schweiz

${ }^{5}$ Klinik für Anästhesie, Intensiv- und Schmerzmedizin, Hessing Kliniken Augsburg, Augsburg, Deutschland

\title{
Ambulante Versorgung akuter Verletzungen der oberen Extremität in der Notfallambulanz in axillärer Plexusanästhesie - Ist das ohne durchgehende Anästhesiebegleitung machbar?
}

Blockaden des Plexus brachialis durch Chirurgen oder Notfallmediziner selbst durchgeführt $[2,4,14]$.

In Deutschland ist dies jedoch üblicherweise Aufgabe des Anästhesisten und soll aufgrund der technikspezifischen Anforderungen wie auch des Nebenwirkungs- und Komplikationsprofils an einem Anästhesiearbeitsplatz durchgeführt werden [10]. Das Integrieren in den allgemeinen Operationsplan führt in der Praxis aber zu Verzögerungen, da innerhalb der Kernarbeitszeit OP- wie Anästhesiekapazitäten meist ausgelastet und außerhalb der Kernarbeitszeit häufig durch Eingriffe höherer Priorität gebunden sind. Lange Wartezeiten sind für Patienten belastend und wirken sich negativ auf die Patientenzufriedenheit oder das Operationsergebnis aus. An unserer Klinik, einem überregionalen Traumazentrum mit ca. 70.000 Visiten/Jahr, werden selektierte Patienten mit kleineren, aber akut operationspflichtigen Verletzungen oder Erkrankungen an Unterarm und Hand chirurgisch außerhalb des allgemeinen Operationsbetriebes in
APB in der Notfallambulanz versorgt. Die APB erfolgt durch Anästhesisten, die weitere operative Versorgung dann ohne weitere Anästhesiebegleitung. Da prinzipiell nicht alle Patienten für ein solches Prozedere geeignet sind, erfordert dies eine gemeinsam mit dem Operateur durchgeführte Patientenselektion. Die Aufgabe des Anästhesisten ist die sichere und verlässliche Durchführung der APB, da die anschließende Operation ohne Überwachung durch Anästhesiepersonal erfolgt. Dies ist eine Herausforderung, da auch nach Einführung der Sonographie APB in einem gewissen Prozentsatz supplementiert oder gar in eine Allgemeinanästhesie konvertiert werden müssen. Es wird in der vorliegenden retrospektiven Analyse anhand von 566 Patienten die mehrjährige Erfahrung mit diesem Behandlungsalgorithmus berichtet.

\section{Methodik}

Patienten mit akut operationspflichtigen Verletzungen oder Erkrankungen an
Der Beitrag beruht teilweise auf einer Posterpr gress am 08.05.2015 in Düsseldorf. 
Tab. 1 Voraussetzungen, Indikationen und Kontraindikationen für die ambulante Versorgung in axillärer Blockade des Plexus brachialis (APB)

Voraussetzungen Kontraindikationen

für die ambulan-

te Versorgung in

ABP

Einwilligung in

beschriebenes

Verfahren

Eingriffe an Hand

und Unterarm

Kein signifikanter Blutverlust zu erwarten

Verletzungen von Beugesehnen, Nerven und Gefäßen sowie Osteosynthesen

Eingriff proximal des Ellenbogens

Hämodynamische Instabilität, respiratorische Insuffizienz oder Überwachungspflicht aus anderen Gründen

Sprachliches und Vigilanzminderung intellektuelles durch Einfluss von AlVerständnis für kohol, Drogen oder das Verfahren und sedierenden Medikaseinen Ablauf mente im Vorfeld

Gute Kommunikation zwischen Operateur und Patient

Unfähigkeit, flach auf dem Rücken liegen zu können (Dyspnoe, Schmerzen, Unruhe)

Hand und/oder Unterarm wurden vom Operateur auf die prinzipielle Eignung für eine operative Versorgung außerhalb des allgemeinen Operationsbetriebes hin untersucht und vorausgewählt.

Kontraindikationen einer operativen Versorgung in der Notaufnahme sind in - Tab. 1 aufgeführt.

Geeigneten Patienten wurde die zeitnahe Versorgung außerhalb des allgemeinen OP-Betriebes in einem aseptischen Eingriffsraum in der Notfallambulanz in APB durch den Operateur angeboten. Die Patienten wurden über das Abweichen von dem allgemeinen Behandlungsalgorithmus informiert. Wesentlicher Bestandteil der Aufklärung war die Erläuterung der operativen Versorgung ohne anästhesiologische Begleitung oder Sedierung. Nach grundsätzlicher Zustimmung des Patienten erfolgte die Prämedikationsvisite durch den Anästhesisten.

Nach grundsätzlicher Zustimmung des Patienten erfolgte die anästhesiologische Vorstellung und Aufklärung. Wurde der Patient von Anästhesist und/ oder Chirurg als nichtgeeignet eingestuft, oder lehnte der Patient ein solches Pro- zedere ab, erfolgte die Versorgung zum nächstmöglichen Zeitpunkt innerhalb des OP-Programms.

War die postoperative Entlassung nach Hause geplant, so wurden den $\mathrm{Pa}$ tienten im Rahmen der Prämedikationsvisite die notwendigen Informationen analog ambulanter Eingriffe gegeben, wie Notwendigkeit einer Begleitperson, Versorgung zu Hause etc. Die Patienten wurden explizit aufgeklärt, dass sie bei einer noch anhaltenden Wirkung der Regionalanästhesie nur in Begleitung nach Hause entlassen werden können, aber bei einer anhaltenden Blockade der betroffenen Extremität besondere Vorsicht zu gelten habe. Als Alternative wurde den Patienten angeboten, das Abklingen der Plexusanästhesie in der Klinik abzuwarten.

Die Patienten wurden ebenfalls aufgeklärt, dass sie sich im Falle einer über $24 \mathrm{~h}$ anhaltenden Wirkung oder sonstiger Auffälligkeiten wie Hämatombildung, Schmerzen an der Punktionsstelle etc., zeitnah in der Notfallambulanz unserer Klinik zur Kontrolle vorstellen sollten.

Die APB wurde sonographisch geführt, unter Monitoring von $\mathrm{EKG}, \mathrm{S}_{\mathrm{p}} \mathrm{O}_{2}$, nichtinvasiver Blutdruckmessung und i.v.-Zugang in der „holding area“ des OPBereiches an einem Anästhesiearbeitsplatz durchgeführt. Die Blockade erfolgte nach dem internen Klinikstandard unter sterilen Kautelen als gezielte selektive Blockade der einzelnen Nerven. Am lateralen Rand des M. pectoralis major wurde die A. axillaris ventral des M. teres major und der Sehneninsertion des M. latissimus dorsi am Humerus identifiziert. Es erfolgte generell die Identifikation der Nervenstämme der 5 den Unterarm und die Hand versorgenden peripheren Nerven des Plexus brachialis (N. radialis, N. medianus, N. musculocutaneus, N. ulnaris, N. cutaneus antebrachii medialis; - Abb. 1).

Nach ultraschallgeführter Hautinfiltration mit 1-2 ml Lidocain, 2\%ig, in Richtung Spatium axillare subfasciale [5] wurden bei Patienten über $60 \mathrm{kgKG}$ initial maximal $30 \mathrm{ml}$ einer Lokalanästhetikamischung von $100 \mathrm{mg}$ Ropivacain, $1 \%$ ig $(10 \mathrm{ml})$, und $400 \mathrm{mg}$ Prilocain, $2 \%$ ig $(20 \mathrm{ml})$, zielgerichtet in einer „Out-of-plane“-Kanülenführung an die entsprechenden Nerven appliziert. Dies entspricht einer Konzentration der Mischlösung von 0,33\%igem Ropivacain und 1,33\%igem Prilocain. Bei Patienten zwischen 50 und $60 \mathrm{kgKG}$ wurde die applizierte Dosis auf $25 \mathrm{ml}$ der Lokalanästhetikamischung begrenzt. Da das Verhältnis von Ropivacain zu Prilocain 1:2 geblieben ist, entspricht die Mischlösung einer Dosis Ropivacain (0,33\%ig) von $82,5 \mathrm{mg}$ und Prilocain (1,66\%ig) von $332,5 \mathrm{mg}$. Dabei wurde besonders auf die zweifelsfreie Identifikation der Kanülenöffnung sowie auf das Ausbreitungsmuster des Lokalanästhetikums während der Injektion geachtet. War ein LAAusbreitungsmuster nach Injektion von maximal $1 \mathrm{ml}$ nicht zweifelsfrei erkennbar, wurden Kanüle und Schallsonde neu zueinander ausgerichtet, bis die Darstellung gelang. Anschließend wurde die Qualität der Blockade sensorisch nach „pinprick“ ausgetestet. War nach 20 min die Blockade unzureichend, so erfolgte eine zielgerichtete selektive Nachblockade der entsprechenden nichtblockierten Nerven mit max. 2-3 ml Prilocain, $1 \%$ ig, und Nerv. Der Patient wurde für $30 \mathrm{~min}$ nach der letzten Injektion in der Holding area unter Fortführung des o.g. Monitoring überwacht. Bei kompletter Blockadewirkung und fehlenden Nebenwirkungen erfolgte nun die chirurgische Versorgung des Patienten in der Notaufnahme ohne weitere anästhesiologische Begleitung. Im Fall unerwünschter Nebenwirkungen wäre der Patient nicht für eine Versorgung außerhalb des OPBereiches freigegeben worden. Die intraoperative Überwachung des wachen Patienten erfolgte durch den Operateur ausschließlich über verbalen Kontakt ohne hämodynamisches oder respiratorisches Monitoring.

Die Datenerfassung erfolgte mittels eines elektronischen Narkoseprotokolls (DANARC $^{\circledR}, \mathrm{R}+\mathrm{S}$ Medical, Hamburg, Deutschland) über die präoperative Verweildauer mit Blockadeanlage und Überwachungsphase in der Holding area. Eine intraoperative anästhesiologische Datenerfassung erfolgte nicht. Für die retrospektive Untersuchung wurden Patienten, die zwischen dem 01.01.2013 und dem 01.12.2017 in APB ohne Anästhe- 
Anaesthesist 2020 • 69:388-396 https://doi.org/10.1007/s00101-020-00772-z

(c) Der/die Autor(en) 2020

A. Rand · C. A. Avila González · G. C. Feigl · T. Mäcken · T. Weiß ·. K. Zahn · R. J. Litz

Ambulante Versorgung akuter Verletzungen der oberen Extremität in der Notfallambulanz in axillärer Plexusanästhesie - Ist das ohne durchgehende Anästhesiebegleitung machbar?

\section{Zusammenfassung}

Hintergrund. Patienten, welche sich mit kleineren, aber akut operationspflichtigen Verletzungen an Hand und/oder Unterarm in der Notaufnahme vorstellen, in den laufenden Operationsbetrieb einzubringen, stellt aufgrund begrenzter Ressourcen eine Herausforderung dar. Eine Alternative bietet die zeitnahe Versorgung in der Notaufnahme. Es wird über die mehrjährige Erfahrung mit einem Behandlungsalgorithmus berichtet, bei dem Notfallpatienten zeitnah in axillärer Plexusblockade (APB) in der Notaufnahme ohne weitere Anästhesiebegleitung versorgt wurden.

Methoden. Patienten wurden auf die prinzipielle Eignung für eine Versorgung außerhalb des allgemeinen Operationsbetriebes vom Operateur vorausgewählt. Nach Ausschluss anästhesiologischer und chirurgischer Kontraindikationen erfolgte in der "holding area" des OP-Bereichs die sonographisch geführte axilläre Plexus-Blockade (APB) in Einzelinjektionstechnik unter Standardmonitoring. Nach Überprüfung der Blockade erfolgte die weitere Versorgung in der Notaufnahme unter Aufsicht des verantwortlichen Chirurgen ohne Anästhesiebegleitung.

Ergebnisse. Von Januar 2013 bis November 2017 wurden 566 Patienten (46,4 J [11-88], $174,9 \mathrm{~cm}$ [140-211], 80,8 kg [42-178], ASA-Status 1/2/3 [190/338/38]) nach dem beschriebenen Behandlungsalgorithmus versorgt. Die APB wurden von 74 Anästhesisten mit maximal $100 \mathrm{mg}$ Ropivacain, $1 \% \mathrm{ig}$, und $400 \mathrm{mg}$ Prilocain, $2 \%$ ig, durchgeführt. Bei $5 \%$ der Patienten war die Blockade nach $20 \mathrm{~min}$ unzureichend, sodass eine Nachinjektion mit maximal 2-3 ml Prilocain, $1 \%$ ig, pro entsprechendem Nerv erfolgte. Danach konnte die chirurgische Versorgung bei allen
Patienten ohne zusätzliche Analgetika oder Nachinfiltrationen durchgeführt werden. Komplikationen der APB wurden nicht beobachtet.

Schlussfolgerung. Wir konnten zeigen, dass die Versorgung ausgewählter Patienten außerhalb des OP mit einem definierten Behandlungsalgorithmus in einer APB sicher und zuverlässig ohne dauerhafte Anästhesiepräsenz möglich war. Unabdingbare Voraussetzungen für ein solches Vorgehen sind aber die sorgfältige Patientenselektion, die Patientenzustimmung, die sichere, effektive Durchführung der APB sowie die verlässliche Absprache mit dem Operateur.

Schlüsselwörter

Axilläre Plexus-brachialis-Blockade . Handverletzungen · Notfallambulanz . Regionalanästhesie · Sonographie

\section{Outpatient treatment of acute injuries of upper extremities with axillary plexus anesthesia in the emergency department-Is that possible without continuous anesthesia attendance?}

\section{Abstract}

Background. The incorporation into the routine operating procedure of patients with small but acute hand and forearm injuries requiring surgery who present in the emergency admission department, represents a challenge due to limited resources. The prompt treatment in the emergency admission department represents an alternative. This article retrospectively reports the authors' experiences with a treatment algorithm in which emergency patients were treated by ultrasound-guided axillary brachial plexus blocks (ABPB) and surgery carried out in the emergency department without further anesthesia attendance.

Methods. Patients were preselected by the surgeon if they were suitable for a standardized treatment without anesthesia attendance during surgery. If there were no anesthesiological or surgical contraindications patients received an $A B P B$ in the holding area of the operating room (OR) under standard monitoring. Blocks were performed as a multiinjection, ultrasound-guided technique which is anatomically described in detail. Patients $>60 \mathrm{~kg}$ received a total volume of $30 \mathrm{ml}$ of a mixture of $10 \mathrm{ml} 1 \%$ ropivacaine $(100 \mathrm{mg})$ and $20 \mathrm{ml} 2 \%$ prilocaine $(400 \mathrm{mg})$. Patients $<60 \mathrm{~kg}$ received the same mixture with a reduced volume of $25 \mathrm{ml}$ corresponding to $82.5 \mathrm{mg}$ ropivacaine and $332.5 \mathrm{mg}$ prilocaine. After controlling for block success patients were admitted to the emergency department and the surgical procedure was carried out under supervision by the surgeon without further anesthesia attendance. At discharge patients were explicitly instructed that in the case of any complications or a continuation of the block for more than $24 \mathrm{~h}$ they should contact the emergency department. Results. Between January 2013 and November 2017 a total of 566 patients (46.4 years, range $11-88$ years, $174.9 \mathrm{~cm}$, range $140-211 \mathrm{~cm}, 80.8 \mathrm{~kg}$, range $42-178 \mathrm{~kg}$, ASA 1/2/3, 190/338/38, respectively) were treated according to a standardized protocol. The ABPBs were performed by 74 anesthetists. In $5 \%$ of the patients the initial block was incomplete and rescue blocks were performed with a maximum of $2-3 \mathrm{ml} 1 \%$ prilocaine per corresponding nerve. After completion the block was ensured and all patients underwent surgery without further analgesics or local anesthetic infiltration by the surgeon. Complications related to the $A B P B$ and readmissions were not observed.

Conclusion. It could be demonstrated that minor surgery could be carried out safely and effectively with a defined algorithm using $A B P B$ in selected patients outside the $O R$ without permanent anesthesia attendance: however, indispensable prerequisites for such procedures are careful patient selection, patient compliance, the safe and effective performance of the ABPB and reliable agreement with the surgeon.

Keywords Emergency department · Axillary brachial plexus block - Hand injuries - Regional anaesthesia $\cdot$ Ultrasound 
Hier steht eine Anzeige.

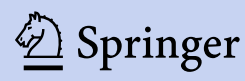




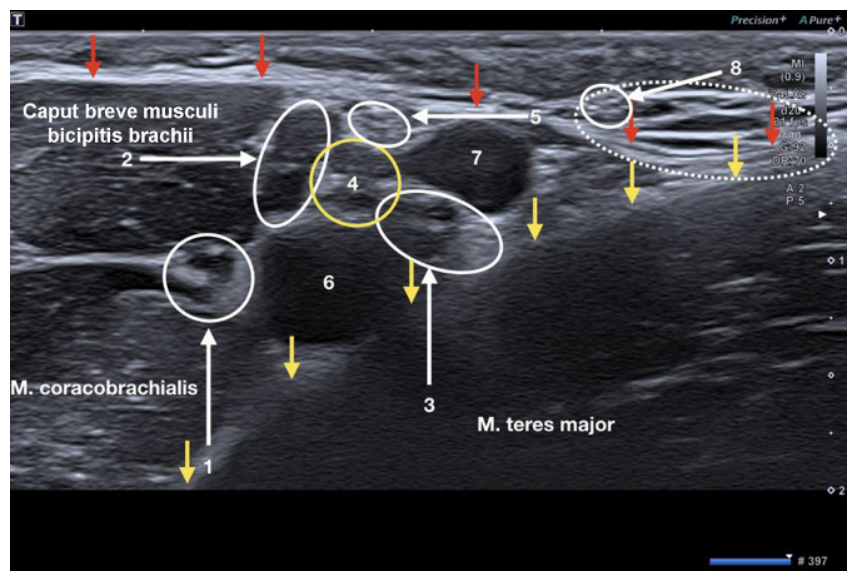

Abb. 1 ॥ Das Sonogramm zeigt die peripheren Nerven des Plexus brachialis in axillärer Position am Rande des M. pectoralis major. $1 \mathrm{~N}$. musculocutaneus, $2 \mathrm{~N}$. medianus, $3 \mathrm{~N}$. radialis, $4 \mathrm{~N}$. ulnaris, $5 \mathrm{~N}$. cutaneus antebrachii medialis, 6 A. axillaris, $7 \mathrm{~V}$. axillaris, $8 \mathrm{~N}$. cutaneus brachii medialis. Der N. cutaneus brachii medialis hat die Plexusloge bereits verlassen und verläuft im Spatium axillare profundus. Rote Pfeile markieren die Fascia axillaris profunda, gelbe Pfeile markieren die Sehne des M. latissimus dorsi mit dem darunter liegenden M. teres major (hypoechogen)

siebegleitung operiert wurden, ausgewertet. Die statistische Analyse erfolgte deskriptiv mit IBM SPSS Statistics ${ }^{\circledR}$ (Version 25, IBM Corp., Armonk NY, USA). Die Daten sind angegeben als Mittelwerte (Range) oder Inzidenzen in Prozent.

\section{Ergebnisse}

Im beschriebenen Beobachtungszeitraum wurden 571 Patienten mit akut operationspflichtigen Verletzungen oder Erkrankungen an Hand oder Unterarm eingeschlossen (• Tab. 2). Fünf Patienten verlangten nach erfolgreicher APB dann entgegen der Absprache doch eine tiefe Sedierung für den operativen Eingriff und mussten folglich in das laufende OP-Programm eingeschoben werden. Diese Patienten wurden aus der weiteren Auswertung ausgeschlossen. Somit konnten 566 Patienten (99\%) nach dem beschriebenen Verfahren versorgt werden (-Tab. 3).

Die Blockaden wurden von insgesamt 74 Anästhesisten je nach Erfahrung allein oder unter Supervision durchgeführt. Die 5 Zielnerven des Plexus brachialis konnten bei allen Patienten identifiziert werden. Bei $95 \%$ der Patienten wurde mit der initialen Blockade eine suffiziente Anästhesie erzielt. Bei $5 \%$ der Patienten war die Blockade nach 20 min nicht ausreichend. Entsprechend wurde gezielt eine zweite Injektion in 3\% der Fälle am
N. musculocutaneus und in jeweils $1 \%$ der Fälle am N. radialis oder am N. ulnaris durchgeführt. Die Nachblockade erfolgte mit maximal $2-3 \mathrm{ml}$ Prilocain $1 \%$ pro Nerv. Danach waren die Blockaden in allen Fällen suffizient. Die geplante chirurgische Versorgung konnte bei allen $\mathrm{Pa}$ tienten ohne zusätzliche Analgetika oder Nachinfiltrationen durch den Operateur durchgeführt werden. In einem Fall erfolgte die Versorgung bei einem 11-jährigen Jungen mit einer Fingerverletzung auf ausdrücklichen Wunsch des Kindes und der Eltern. In diesem Fall wurden $3 \mathrm{ml}$ Ropivacain, 0,5\%ig, und $6 \mathrm{ml}$ Prilocain, $1 \%$ ig, entsprechend dem Klinikstandard für Blockaden bei Kindern unter 14 Jahren verwendet. Der Junge wurde während der gesamten Prozedur von den Eltern begleitet. Bei einem Patienten mit einem Körpergewicht $>130 \mathrm{~kg}$ war die Blockade nach initialem Wirkungseintritt innerhalb 30 min komplett rückläufig, sodass mit der halben Dosis nachinjiziert wurde. Nach einer weiteren unauffälligen Beobachtungszeit von 30 min wurde der Patient für die Versorgung freigegeben. Der weitere Verlauf war unauffällig. Punktionskomplikationen oder Zeichen einer Lokalanästhetikaintoxikation während der Injektion, der anschließenden Überwachung oder der chirurgischen Versorgung wurden nicht beobachtet. In keinem Fall kam es infolge von Komplikationen des An- ästhesieverfahrens oder einer nach Entlassung über $24 \mathrm{~h}$ anhaltenden Blockadewirkung zu einer Wiedervorstellung in der Ambulanz.

\section{Diskussion}

Wir konnten zeigen, dass eine zügige und sichere Versorgung von Notfallpatienten mit klar definierten akuten Verletzungen oder Erkrankungen an Hand und Unterarm in APB ohne Zeitverzögerung auch ohne dauerhafte Anästhesiepräsenz außerhalb des regulären OPBetriebes möglich ist. Hierbei sind jedoch zwingend mehrere Voraussetzungen zu berücksichtigen.

Zum einen muss die APB zuverlässig und komplikationslos durchgeführt werden, wobei zu beachten ist, dass auch nach Einführung der Sonographie in einem gewissen Prozentsatz eine Supplementierung oder gar Konversion zur Allgemeinanästhesie erforderlich sein kann. Weiterhin muss der Patient seitens seiner Komorbidität und Compliance für ein solches Verfahren geeignet sein und diesem zustimmen. Da prinzipiell nicht alle Patienten für ein solches Vorgehen infrage kommen, erfordert dies eine gemeinsam mit dem Operateur durchgeführte sorgfältige Patientenselektion. Der Patient muss das Verfahren und seine Besonderheiten intellektuell verstehen. Darüber hinaus muss vor und während des Eingriffs jederzeit eine barrierefreie Verständigung zwischen Patient und Operateur möglich sein. $\mathrm{Zu}$ Beginn muss sich der Operateur von der Eignung des Patienten für ein solches Vorgehen überzeugen, endgültig entscheidet jedoch der Anästhesist über die Machbarkeit. Ist der Patient ängstlich oder unruhig, so ist er für ein solches Verfahren nicht geeignet, da während der OP planmäßig kein Anästhesist für eine Sedierung und die damit erforderliche Überwachung der Vitalfunktionen bereitsteht. Wird eine Sedierung erforderlich, muss in jedem Fall ein Anästhesist hinzugezogen und bis zu dessen Verfügbarkeit der Eingriff ggf. unterbrochen werden. Daher wurden Patienten, die vor der Anästhesievorstellung in der Notaufnahme Opioide oder Sedativa erhalten oder Drogen und Alkohol konsumiert hatten, wegen nichtkal- 


\section{Tab. 2 Art der Verletzungen oder Erkrankungen}

\begin{tabular}{lll}
\hline Diagnose & Anzahl $(\boldsymbol{n}=\mathbf{5 6 6})$ & Prozent \\
\hline $\begin{array}{l}\text { Schnitt-/Quetschverletzungen der Hand und des Handgelenks } \\
\text { Infektionen der Weichteile an Hand und Unterarm }\end{array}$ & 194 & 34,2 \\
\hline $\begin{array}{l}\text { Schnitt-/Quetschverletzungen am Unterarm/geschlossene } \\
\text { Repositionen }\end{array}$ & 156 & 27,6 \\
\hline $\begin{array}{l}\text { Nervenläsionen und Neuropathien } \\
\text { Hämarthros des Handgelenks }\end{array}$ & 33 & 6,5 \\
\hline $\begin{array}{l}\text { Verbrennungen } \\
\text { Sonstiges }\end{array}$ & 31 & 5,8 \\
\hline Gesamt & 9 & 5,5 \\
\hline & 106 & 1,6 \\
\hline & 566 & 18,7 \\
\hline
\end{tabular}

kulierbarer Compliance von dem Verfahren ausgeschlossen. In dieser Untersuchung wurde im Fall der 5 Patienten, die doch auf einer intraoperativen Sedierung bestanden, entsprechend verfahren. Hämodynamisch oder respiratorisch instabile Patienten oder Patienten, die aus anderen Gründen ein kontinuierliches Monitoring benötigen, sind für ein solches Vorgehen nicht geeignet. Ebenso wenig sind Patienten geeignet, die für die Dauer des Eingriffs nicht ruhig liegen können. Daher beschränkte sich das hier beschriebene Verfahren auf Patienten, die alle diese geforderten Kriterien erfüllten. Zuletzt muss sich der Operateur sicher sein, den Eingriff in alleiniger APB durchführen zu können, da ein Umstieg auf ein anderes Anästhesieverfahren ohne signifikante Zeitverzögerung nicht möglich ist. Daher ist neben der kritischen Prüfung der Indikation eine eindeutige Absprache zwischen Anästhesist und Operateur über das geplante Vorgehen erforderlich.

Grundsätzlich sind bei der Durchführung von Regionalanästhesieverfahren die allgemeinen Sicherheitsstandards einzuhalten. Hierzu gehört zweifelsohne die Überwachung bei der Blockadeanlage selbst, sowie im weiteren Verlauf bis zum vollständigen Wirkungseintritt. Die weitere intraoperative Überwachung erfolgte in unserer Untersuchung durch den Operateur.

Es existiert eine gültige Stellungnahme des Wissenschaftlichen Arbeitskreises Regionalanästhesie von 1997 zur Frage der Durchführung von Regionalanästhesien durch Operateure mit klaren Empfehlungen [10], welche sich auf Lokal- und Regionalanästhesien be- ziehen, bei deren Durchführung keine Auswirkungen auf vitale Funktionen zu erwarten sind. Dies sind v. a. die Infiltration des Operationsgebietes oder eine operationsfeldnahe Regionalanästhesie, wie die Leitungsanästhesie nach Oberst. Somit scheiden rückenmarknahe Leitungsverfahren aufgrund des Risikos gravierender, auch mit Latenz auftretender, hämodynamischer Nebenwirkungen für eine solche Vorgehensweise aus [11]. Plexusanästhesien oder andere proximale Nervenblockaden wie N.-femoralis-/N.-ischiadicus-Blockaden weisen durch die höheren Lokalanästhetikadosierungen das Risiko einer systemischen Lokalanästhetikatoxizität auf und erfordern daher sichere Kenntnisse in deren frühzeitigen Erkennung und ggf. Behandlung. Folglich ist auch hier prinzipiell eine sachgerechte dauerhafte Überwachung zu gewährleisten.

International werden solche peripheren Regionalanästhesieverfahren jedoch in zunehmendem Maße in den Notfalldepartments durch Notfallmediziner ohne Anästhesieweiterbildung und nicht durch Anästhesisten durchgeführt [2, 18].

Diese Verfahren beinhalten sowohl die Leitungsanästhesie einzelner Nerven an Arm und Bein, aber auch Interkostal- oder M.-erector-spinae-Blockaden sowie Plexusanästhesien $[3,6,12,13,17$, 22].

Während Allgemeinanästhesieverfahren eindeutig dem Fachgebiet vorbehalten sind, lässt sich bezüglich anderer Regionalanästhesieverfahren ein Ausschließlichkeitsanspruch, den das Bundesverfassungsgericht ohnehin nur
Tab. 3 Patientencharakteristika und ver-

wendete Lokalanästhetikadosierung (Ropi-

vacain $0,66 \%$ und Prilocain $1,33 \% / \mathrm{ml}$ ). Die

Daten sind angegeben als Mittelwerte und

Standardabweichung (Range)

\begin{tabular}{l|l}
\hline Patientencharakteristika & $\mathbf{n = 5 6 6}$ \\
\hline Geschlecht (m/w) & $387 / 179$ \\
\hline $\begin{array}{l}\text { Alter (Jahre) } \\
\text { Größe }(\mathrm{cm})\end{array}$ & $46,4(11-88)$ \\
\hline $\begin{array}{l}174,9 \\
(140-211)\end{array}$ \\
\hline Körpergewicht (kg) & $80,8(42-178)$ \\
\hline $\begin{array}{l}\text { ASA-Status 1/2/3 } \\
\text { Lokalanästhetikavolumen } \\
\text { (ml) }\end{array}$ & $190 / 338 / 38$ \\
\hline
\end{tabular}

eingeschränkt gewährt, für das Fachgebiet nicht ableiten. Dennoch gilt, bei Verfahren, die ihren Schwerpunkt im Fachgebiet Anästhesiologie haben, den Facharztstandard einzuhalten. Eine ähnliche Arbeitsteilung ist in der $\mathrm{Zu}$ sammenarbeit mit Geburtshelfern in den Empfehlungen zur Zusammenarbeit mit anderen Fachgebieten unter Ziffer 4.1. geregelt. Hier legt der Anästhesist die Regionalanästhesie (Epiduralanästhesie) an, die dann von einem anderen Fachvertreter, dem Geburtshelfer, weitergeführt werden kann. Voraussetzung ist, dass die Reaktionen der Patientin auf die epidural applizierten Substanzen erfasst und dokumentiert sind. Dann kann sogar die Durchführung weiterer Bolusapplikationen zur Aufrechterhaltung der Analgesie nach ärztlicher Anordnung an entsprechend ausgebildetes medizinisches Personal delegiert werden. Weitere Voraussetzungen sind, dass der durchführende Arzt sich von der Kompetenz und Fähigkeit des Delegaten überzeugt hat und sich die delegierte Person selbst als kompetent und fähig im Umgang mit dem Verfahren einstuft [1].

Unsere Vorgehensweise unterscheidet sich in wesentlichen Punkten von den in Notfalldepartments üblichen Abläufen. Die APB wurde nicht von Operateuren oder Notfallmedizinern durchgeführt, sondern von Anästhesisten unter Einhaltung allgemeiner Sicherheitsstandards sowie unter Monitoring der Vitalparameter. Die Patienten wurden erst nach einer 30-minütigen Überwachungsphase dem Operateur zur weiteren Betreuung und Versorgung übergeben. 


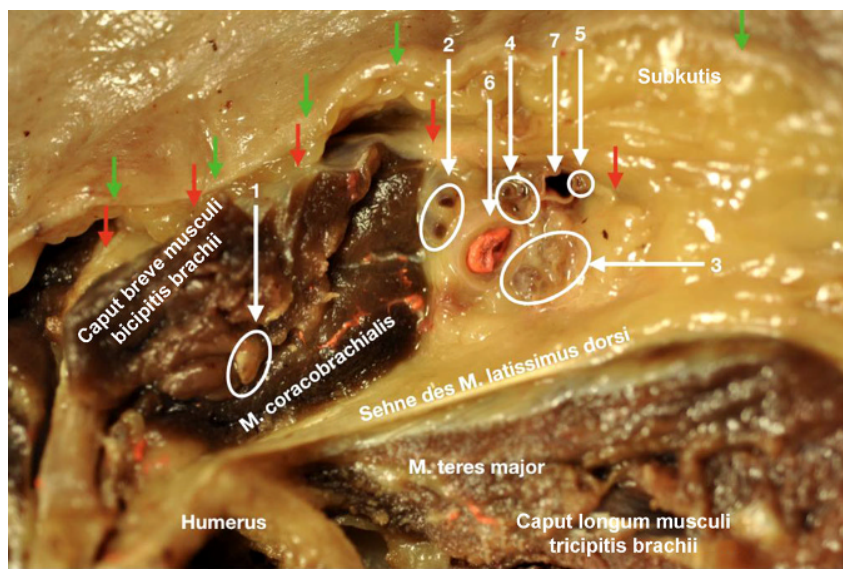

Abb. $2 \Delta$ Präparat des Plexus brachialis in axillärer Position zeigt im Querschnitt die peripheren Nerven des Plexus brachialis mit ihren Bindegewebshüllen und dazwischen liegendem Fettgewebe sowie die Beziehung zu Muskeln und Faszien. $1 \mathrm{~N}$. musculocutaneus, $2 \mathrm{~N}$. medianus, $3 \mathrm{~N}$. radialis, $4 \mathrm{~N}$. ulnaris, $5 \mathrm{~N}$. cutaneus antebrachii medialis, $6 \mathrm{~A}$. axillaris mit Latexfüllung 7 V. axillaris. Rote Pfeile markieren die Fascia axillaris profunda, grüne Pfeile die Kutis

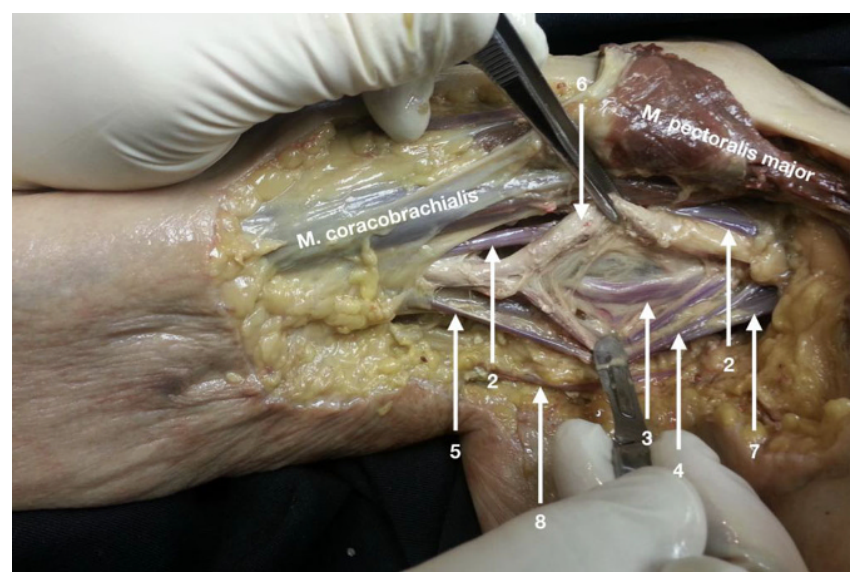

Abb. $3 \Delta$ Das Präparat des Plexus brachialis in axillärer Position am Rande des M. pectoralis major auf Höhe des Sehnenansatzes des M. latissimus dorsi am Humerus zeigt die peripheren Nerven des Plexus brachialis mit ihren Bindegewebshüllen und dazwischen liegendem Fettgewebe. Der M. pectoralis major ist durchtrennt und angehoben. Der N. musculocutaneus (in - Abb. 2 mit 1 bezeichnet) ist hier nicht sichtbar, 2 N. medianus, $3 \mathrm{~N}$. radialis, $4 \mathrm{~N}$. ulnaris, $5 \mathrm{~N}$. cutaneus antebrachii medialis, $6 \mathrm{~A}$. axillaris, $7 \mathrm{~V}$. axillaris, $8 \mathrm{~N}$. intercostobrachialis
Neben wirtschaftlichen Überlegungen sowie der Forderung der Patienten nach einer zeitnahen Versorgung spielt sicher die Einführung der Sonographie in die periphere Regionalanästhesie (RA) eine wesentliche Rolle, um über solche Verfahrensweisen nachzudenken. Die grundsätzlichen Vorteile der sonographischen RA wie die Darstellung der Punktionskanüle, die Beurteilbarkeit der Lokalanästhetikaausbreitung während der Injektion und das Erkennen anatomischer (Gefäß-)Variationen im Punktionssitus sind mittlerweile allgemein anerkannt. Da sonographisch die intravasale Fehlinjektion bereits kleinster Volumina sicher erkannt werden kann, ist die Gefahr von Intoxikationen hierdurch als sehr gering zu bewerten. Es bleibt jedoch ein Restrisiko durch Resorption der Lokalanästhetika, dessen Ausmaß in erster Linie von der Spitzenkonzentration im Plasma und der Geschwindigkeit ihres Anstiegs abhängt und maßgeblich durch den Injektionsort bestimmt wird [7, 16]. Anhand der APB konnte für verschiedene LA gezeigt werden, dass im Regelfall die höchsten Plasmakonzentrationen nach 20-30 min, vereinzelt jedoch Spitzenkonzentrationen auch erst nach 60 min erreicht werden $[15,21]$. Intoxikationen, die erst zwischen 30 und
60 min auftraten, wurden hierbei aber nicht berichtet.

Aus diesem Grund wurden die Patienten für mindestens 30 min nach Injektion überwacht und nur bei völliger Symptomfreiheit für den Eingriff freigegeben.

Mit einer Ausnahme wurden bei normalgewichtigen Erwachsenen Dosierungen von maximal $400 \mathrm{mg}$ Prilocain und $100 \mathrm{mg}$ Ropivacain verwendet. Da es keine wissenschaftlich genau belegbaren Maximaldosierungen gibt und sich die Empfehlungen noch länderspezifisch signifikant unterscheiden, sollten die verwendeten Dosierungen regionalanästhesiespezifisch ausgerichtet werden und neben dem Injektionsort patientenbezogene Faktoren wie Alter, Komorbidität und Schwangerschaft miteinbeziehen [19]. Da solche Faktoren die Pharmakokinetik der LA wesentlich mitbestimmen, wurden in unserer Untersuchung nur nichtschwangere Patienten bis zum ASA-Status 3 eingeschlossen. Zusätzlich wurden die für Deutschland empfohlenen Maximaldosierungen nicht überschritten. Diese sind für Prilocain ohne Adrenalinzusatz $600 \mathrm{mg}$ und für Ropivacain $300 \mathrm{mg}$ für Plexusbrachialis-Blockaden, bezogen auf Erwachsene mit $70 \mathrm{kgKG}$. Die in unserem Vorgehen beschriebene Überwachungs- zeit unter Monitoring von $30 \mathrm{~min}$ nach Beendigung der Injektion erwies sich als ausreichend für die Erfassung von evtl. Intoxikationszeichen.

Ein klinisches Problem der APB ist auch nach Einführung der Sonographie weiterhin die Rate an inkompletten Blockaden oder gar Blockadeversagern. Selbst für die anatomisch fundierte Multiinjektionstechnik ist beschrieben, dass in einem gewissen Prozentsatz Supplementierungen oder Überleitungen in eine Allgemeinanästhesie erforderlich sind [8]. Die Sicherstellung der Analgesie ist somit eine der zentralen Anforderungen an den Anästhesisten. Aus anatomischer Sicht ist die APB eine Einzelnervenblockade und keine Plexusblockade per se, da in axillärer Position die die Hand und den Unterarm versorgenden Nerven aus ihren entsprechenden Faszikeln oder Trunci bereits abgegangen sind und im weiteren Verlauf getrennt in eigenen Bindegewebstunneln, eingebettet in Fettgewebe, verlaufen (๑Abb. 2 und 3). Aus diesen anatomischen Gründen können Einzel- oder Doppelinjektionen alle Zielnerven in ihren einzelnen Kompartimenten nicht zuverlässig erreichen.

Aufgrund dieser Tatsachen führen wir die APB gemäß dem Klinikstandard sonographisch als Einzelinjektionstechnik an den 5 Einzelnerven durch. Der Vor- 
schub der Punktionskanüle sowie die LAInjektion erfolgen unter sicherer sonographischer Bildgebung. Dies gilt auch für die subkutane Infiltration vor der eigentlichen Einführung der Punktionskanüle. Diese Infiltration wird ebenfalls ultraschallgesteuert und bis auf die Oberarmfaszie durchgeführt, um den N. intercostobrachialis, welcher an dieser Stelle regelhaft im Spatium axillare subfasciale verläuft, zu erreichen [5]. Mit dieser Vorgehensweise konnten signifikante intravasale oder intramuskuläre Fehlinjektionen, die zu hohen Serumkonzentrationen der LA führen können, vermieden werden [23]. Diese zielgerichtete Einzelinjektionstechnik in die entsprechenden Kompartimente erklärt die hohe primäre Erfolgsrate [9].

Dennoch war in $5 \%$ der Fälle eine Nachinjektion erforderlich, die aber in allen Fällen zu einer ausreichenden Blockade führte. Das Risiko einer LAIntoxikation kann am ehesten durch Verwendung der geringstmöglichen Dosis, einer kontrollierten ultraschallgesteuerten Injektion sowie einer umfassenden Ausbildung der durchführenden Ärzte minimiert werden. In der hier beschriebenen Technik ist die APB faktisch eine Leitungsanästhesie von 5 nah beieinander liegenden, aber in getrennten Bindegewebskompartimenten verlaufenden, peripheren Nerven. Die Stellungnahme des Wissenschaftlichen Arbeitskreises Regionalanästhesie sieht vor, dass Infiltrations- oder periphere Leitungsanästhesien auch durch nichtanästhesiologisches ärztliches Personal durchgeführt werden können. Wenn hier auch primär Verfahren wie z.B. die Leitungsanästhesie nach Oberst gemeint sind, bei der die anatomisch weit peripher gelegenen Nn. digitales palmares oder Nn. digitales plantares proprii anästhesiert werden, so sollte dies, anatomisch betrachtet, auch auf zentraler gelegene Nerven, wie z.B. N. radialis oder N. medianus übertragbar sein, ohne das Risiko von Komplikationen zu erhöhen. Um die notwendige Sicherheit und Erfolgsrate zu gewährleisten, empfehlen die Autoren jedoch, auch im Hinblick auf die verwendeten Lokalanästhetikadosen, die Durchführung des beschriebenen Verfahrens regionalanäs- thesiologisch geschulten Anästhesisten vorzubehalten [20].

Zusammenfassend konnten wir zeigen, dass die Versorgung ausgewählter Patienten außerhalb des OP mit einem standardisierten Verfahren in einer APB sicher und zuverlässig auch ohne eine dauerhafte Anästhesiepräsenz möglich ist. Somit kann die Versorgung zeitkritischer Patienten zügig und sicher unter bedarfsgerechtem Einsatz begrenzter OP- und Personalressourcen erzielt werden. Unabdingbare Voraussetzungen dafür sind aber die sorgfältige Patientenselektion und Zustimmung des Patienten, die sichere Durchführung der APB durch erfahrene Anästhesisten sowie die absolut verlässliche Absprache mit dem Operateur.

\section{Korrespondenzadresse}

\section{Dr. A. Rand}

Klinik für Anästhesiologie und Intensivmedizin, Universitätsklinikum Carl Gustav Carus, TU Dresden

Fetscherstr. 74, Dresden, Deutschland

axel.rand@uniklinikum-dresden.de

Funding. Open Access funding provided by Projekt DEAL.

\section{Einhaltung ethischer Richtlinien}

Interessenkonflikt. A. Rand, C. Avila González, G. C. Feigl, T. Mäcken, T. Weiß, P. K. Zahn und R. J. Litz geben an, dass kein Interessenkonflikt besteht.

Für diesen Beitrag wurden von den Autoren keine Studien an Menschen oder Tieren durchgeführt. Für die aufgeführten Studien gelten die jeweils dort angegebenen ethischen Richtlinien.

Open Access. Dieser Artikel wird unter der Creative Commons Namensnennung 4.0 International Lizenz veröffentlicht, welche die Nutzung, Vervielfältigung, Bearbeitung, Verbreitung und Wiedergabe in jeglichem Medium und Format erlaubt, sofern Sie den/die ursprünglichen Autor(en) und die Quelle ordnungsgemäß nennen, einen Link zur Creative Commons Lizenz beifügen und angeben, ob Änderungen vorgenommen wurden.

Die in diesem Artikel enthaltenen Bilder und sonstiges Drittmaterial unterliegen ebenfalls der genannten Creative Commons Lizenz, sofern sich aus der Abbildungslegende nichts anderes ergibt. Sofern das betreffende Material nicht unter der genannten Creative Commons Lizenz steht und die betreffende Handlung nicht nach gesetzlichen Vorschriften erlaubt ist, ist für die oben aufgeführten Weiterverwendungen des $\mathrm{Ma}$ terials die Einwilligung des jeweiligen Rechteinhabers einzuholen.
Weitere Details zur Lizenz entnehmen Sie bitte der Lizenzinformation auf http://creativecommons.org/ licenses/by/4.0/deed.de.

\section{Literatur}

1. BDA, DGAI (2009) 2. überarbeitete Empfehlungen der Deutschen Gesellschaft für Anästhesiologie und Intensivmedizin und des Berufsverbandes Deutscher Anästhesisten in Zusammenarbeit mit der Deutschen Gesellschaft für Gynäkologie und Geburtshilfe (2009):Durchführung von Analgesieund Anästhesieverfahren in der Geburtshilfe. Anaesthesiol Intensivmed 50:490-495

2. Bhoi S, Sinha TP, Rodha M, Bhasin A, Ramchandani R, Galwankar S (2012) Feasibility and safety of ultrasound-guided nerve block for management of limb injuries by emergency care physicians. JEmerg Trauma Shock 5:28-32

3. Blaivas M, Adhikari S, Lander L (2011) A prospective comparison of procedural sedation and ultrasound-guided interscalene nerve block for shoulder reduction in the emergency department. Acad Emerg Med 18:922-927

4. De Buck F, Devroe S, Missant C, Van de Velde M (2012) Regional anesthesia outside the operating room: indications and techniques. Curr Opin Anaesthesiol 25:501-507

5. Feigl G, Aichner E, Mattersberger C, Zahn PK, Avila Gonzalez C, Litz R (2018) Ultrasound-guided anterior approach to the axillary and intercostobrachial nerves in the axillary fossa: an anatomical investigation. Br J Anaesth 121:883-889

6. Flores S, Herring AA (2016) Ultrasound-guided greater auricular nerve block for emergency department ear laceration and ear abscess drainage. JEmerg Med 50:651-655

7. Griffiths JD, Le NV, Grant S, Bjorksten A, Hebbard P, Royse C (2013) Symptomatic local anaesthetic toxicity and plasma ropivacaine concentrations after transversus abdominis plane block for Caesarean section. Br J Anaesth 110:996-1000

8. Imasogie N, Ganapathy S, Singh S, Armstrong K, Armstrong P (2010) A prospective, randomized, double-blind comparison of ultrasound-guided axillary brachial plexus blocks using 2 versus 4 injections. Anesth Analg 110:1222-1226

9. Janzen PR, Vipond AJ, Bush DJ, Hopkins PM (2001) A comparison of $1 \%$ prilocaine with $0.5 \%$ ropivacaine for outpatient-based surgery under axillary brachial plexus block. Anesth Analg 93:187-191

10. Klose RHV, Wulf $\mathrm{H}$ et al (1996) Zur Frage der Durchführung von Regionalanästhesien durch Operateure. Anasth Intensivmed 37:412-413

11. Litz RJ, Koch T (2007) Steuerbarkeit der Spinalanästhesie - nach wie vor ein ungelöstes Problem? Anasth Intensivmed 48:404-418

12. Luftig J, Mantuani D, Herring AA, Dixon $B$, Clattenburg E, Nagdev A (2018) Successful emergency pain control for posterior rib fractures with ultrasound-guided erector spinae plane block. Am JEmerg Med 36:1391-1396

13. Lyons C, Herring AA (2017) Ultrasound-guided axillary nerve block for $E D$ incision and drainage of deltoid abscess. Am J Emerg Med 35:1032.e3-1032.e7

14. Mackay CA, Bowden DF (1997) Axillary brachial plexus block-an underused technique in the accident and emergency department. J Accid Emerg Med 14:226-229 


\section{Originalien}

15. Maclean D, Chambers WA, Tucker GT, Wildsmith JA (1988) Plasma prilocaine concentrations after three techniques of brachial plexus blockade. $\mathrm{Br} J$ Anaesth 60:136-139

16. Müller M, Litz RJ, Hübler M, Albrecht DM (2001) Grand mal convulsion and plasma concentrations after intravascular injection of ropivacaine for axillary brachial plexus blockade. Br J Anaesth 87:784-787

17. Raeyat DoostE, Heiran MM, Movahedi M, Mirafzal A (2017) Ultrasound-guided interscalene nerve block vs procedural sedation by propofol and fentanyl for anterior shoulder dislocations. Am J Emerg Med 35:1435-1439

18. Reid N, Stella J, Ryan M, Ragg M (2009) Use of ultrasound to facilitate accurate femoral nerve block in the emergency department. Emerg Med Australas 21:124-130

19. Rosenberg PH, Veering BT, Urmey WF (2004) Maximum recommended doses of local anesthetics: a multifactorial concept. Reg Anesth Pain Med 29:564-575 (discussion 524)

20. Savoia G, Coluzzi F, Di Maria C et al (2015) Italian intersociety recommendations on pain management in the emergency setting (SIAARTI, SIMEU, SIS 118, AISD, SIARED, SICUT, IRC). Minerva Anestesiol 81:205-225

21. Simon MA, Vree TB, Gielen MJ, Booij LH, Lagerwerf AJ (1998) Comparison of the effects and disposition kinetics of lidocaine and (+/-)prilocaine in patients undergoing axillary brachial plexus block during day case surgery. Clin Drug Investig 16:241-250

22. Tezel O, Kaldirim U, Bilgic S, Deniz S, Eyi YE, Ozyurek S, Durusu M, Tezel N (2014) A comparison of suprascapular nerve block and procedural sedation analgesia in shoulder dislocation reduction. Am J Emerg Med 32:549-552

23. Yamamoto K, Nomura T, Shibata K, Ohmura S (1997) Failed axillary brachial plexus block techniques result in high plasma concentrations of mepivacaine. Reg Anesth 22:557-561

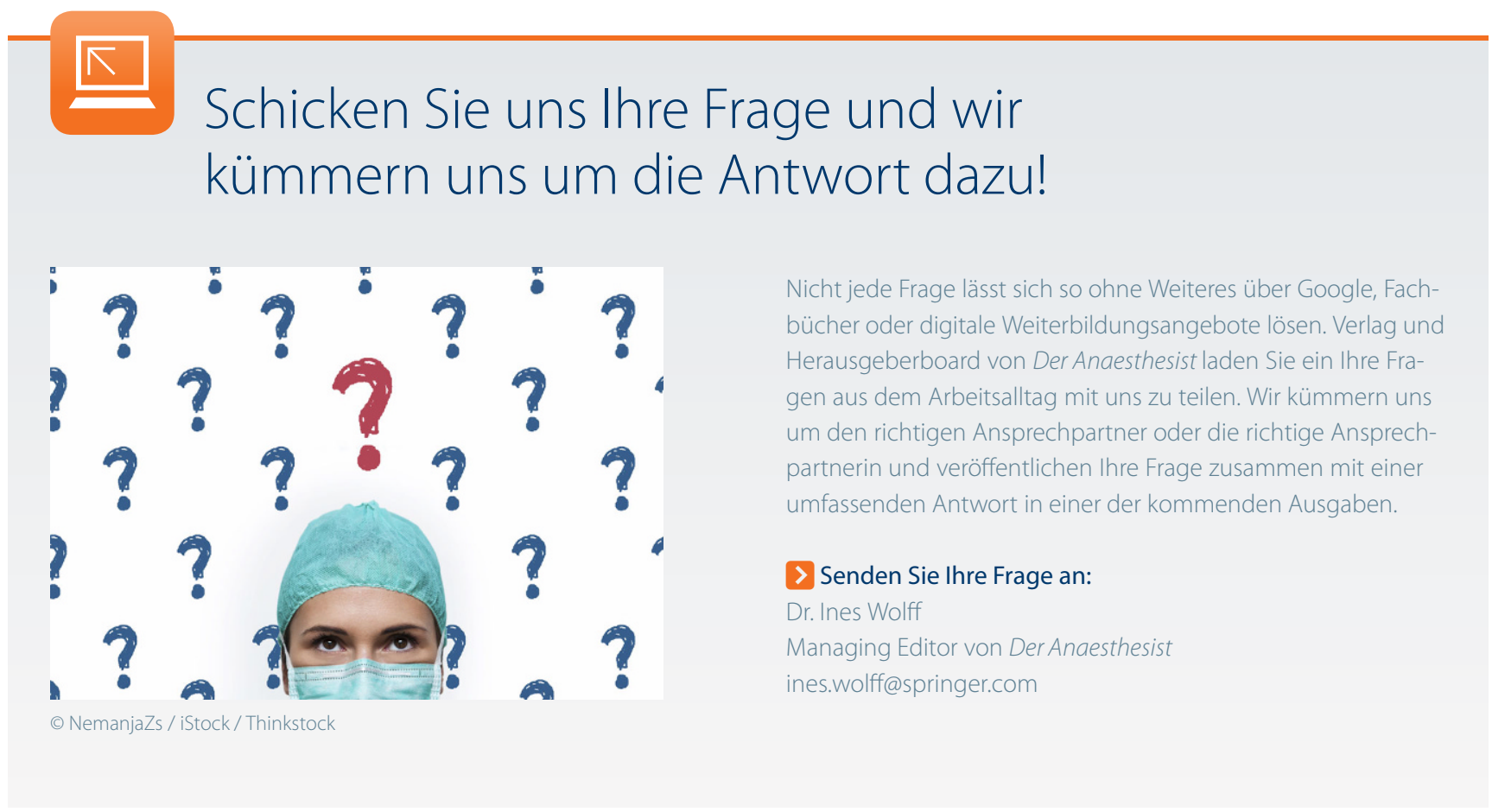

